\title{
A Study on the Simplified Scheme for the Assessment of the Welding-induced Buckling Sensitivity in Thin Plate Panels
}

\author{
Myoung Soo Han*, \\ *Daewoo Shipbuilding \& Marine Eng. Co., Ltd., Geoje, 53302, Korea \\ †Corresponding author : mshan@dsme.co.kr \\ (Received March 25, 2020 ; Revised April 21, 2020 ; Accepted May 11, 2020)
}

\begin{abstract}
The welding-induced buckling distortion is one of the prime concerns in the welded thin plate panels. In this study, an analytic scheme of the plasticity-based elastic buckling prediction was proposed to assess the welding-induced buckling sensitivity of the thin plate panels. Through the scheme, the effect of the structural design and welding fabrication variables on the buckling sensitivity has been analytically investigated. Experiments were also conducted to observe the welding distortion in the test panels. It was found that buckling was unavoidable unless the heat input to the thin plates during butt welding was adequately controlled. It was noted that the buckling distortion in thin plate panels may not be satisfactorily prevented either by considering only the structural arrangement or by controlling only the welding variables. Therefore, the integrated structural design is required in addition to the consideration of welding parameters for the panel assembly. Under those circumstances, it is expected that the proposed scheme can be an effective measure to identify whether an integrated structural and welding design for the thin plate panels can be put in production or if it should be withdrawn for further engineering consideration.
\end{abstract}

Key Words : Welding-induced buckling distortion, Thin plate panel, Buckling sensibility, Eigenvalue analysis

\section{Introduction}

The panel, which is the plate orthogonally stiffened with longitudinal and transverse stiffeners, is the principal component of ship structures. Welded panels accumulate the distortion due to the repeated thermal cycles during the multiple welding assembly stages. The magnitude of distortion depends on complex design and fabrication parameters, including the welding heat input, the plate thickness, the structural stiffness, the fit-up accuracy, and the assembling sequence. In the fabrication of thin plate panels, an unacceptable level of the out-of-plane distortion can often be generated, and thus lots of the correction work should be costly implemented at the subsequent assembling stage. Among the types of the out-of-plane distortion, welding-induced buckling is known as the dominant source of dimensional inaccuracy in thin plate panels ${ }^{1,2)}$. As high strengthened steels and aggressive design optimization in recent years has promoted a wide use of thinner plates, the welding-induced buckling becomes a major concern of the thin plate structures.

Indeed, excessive buckling distortion must be avoided because once the buckling distortion occurs during the fabrication stages, the panels can hardly maintain the flatness within a tolerant level. It makes the subsequent assembling processes suspended. In order to prevent the buckling distortion, it is desirable to minimize the welding heat input for every welded joint ${ }^{3,4)}$. It has been reported that intermittent welding and optimal welding sequences are effective on alleviating the distortion ${ }^{5-7)}$. Mitigation schemes accompanying restraints, additional thermal control have been proposed to prevent the excessive distortion where supplemental heating and cooling measures during welding were utilized ${ }^{8,9}$. Some measures were devised to exploit the opposing forces to reduce the welding residual stresses ${ }^{5)}$.

Despite more costly rework to rectify the distortion, however, the post-welding correction work appears to be more common practice in the commercial shipbuilding. 
It is partly because those preventive measures may not be ordinary enough to be fit with traditional panel fabrication lines. In addition, the adoption of those measures, being typically effective on the thin plates, may cause unpredictable disruption to the well-ordered existing production lines. As an alternative solution to control the buckling distortion in welded thin plates, the laser welding processes have been actively introduced to minimize the welding heat input ${ }^{10,11)}$. It is deemed, however, that the production system equipping the laser facility may demand a large scale of the capital expenditure whereas there exists the concern about the lack of productivity especially when the system should cope with the structural components of which thickness is not thin.

With the advancement in three-dimensional (3D) finite element analyses in recent years, the numerical prediction capability has been remarkably improved in various engineering fields. The application of the 3D analysis to the large welded structures is, however, prone to be unsatisfactory so far since a feasible 3D analysis procedure may not easily be found due to extensive computational time and limited resources available.

In this study, a simplified analytical scheme was proposed to assess the fitness of the design and the assembly procedure to minimize the possibility of the welding-induced buckling distortion in thin plate panels. With the scheme, the buckling sensitivity of the panels was predicted in connection with the structural design modification, the different levels of the welding heat inputs, and the assembling stages. Experiments were also carried out to observe the magnitude and the mode of the welding-induced buckling distortion for the cases that resulted in the buckling instability from the analytical prediction.

\section{Prediction of the Buckling Sensitivity}

\subsection{Assembly procedure of the thin plate panels}

Fig. 1 shows the configuration and dimension of the thin plate panel. The welded panel consists of the top plate, the longitudinal stiffeners, and the transverse frames. The $6 \mathrm{~mm}$ thick top plate is $4830 \mathrm{~mm}$ in length and $(3240+2 w) \mathrm{mm}$ in width, depending on the width of the free edge, $w$, on both sides. The longitudinal stiffener is the angle, and the transverse frame is the T-shaped built-up beam. Unlike the ordinary longitudinal stiffeners, an H-shaped one is placed in the middle of the panel to back the root of the butt weld.

Fig. 2 illustrates the assembly procedure for the panel. After fitting up the longitudinal stiffeners at a regular

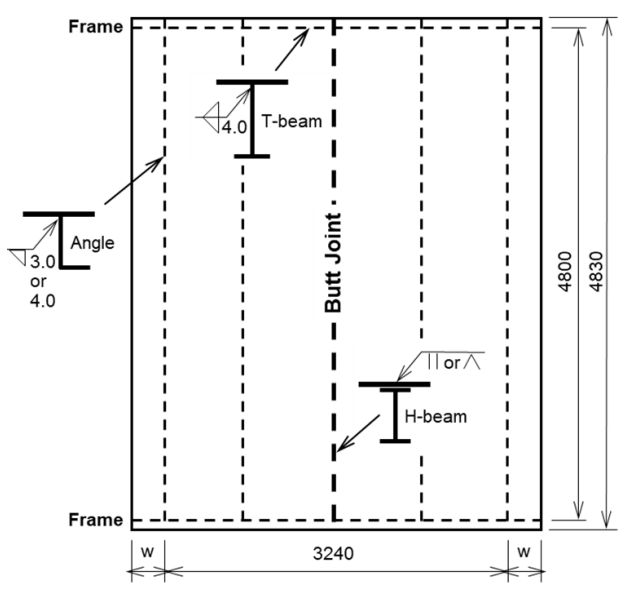

Fig. 1 Configuration and dimension of the panel

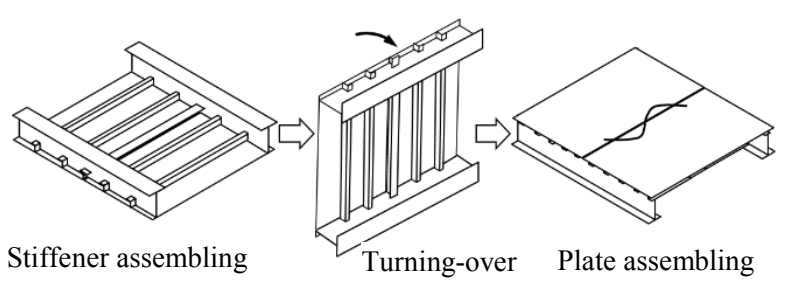

Fig. 2 Assembling sequence of the panel

Table 1 Fillet welding conditions

\begin{tabular}{|c|c|c|c|c|}
\hline Process & $\begin{array}{c}\text { Throat } \\
(\mathrm{mm})\end{array}$ & $\begin{array}{c}\text { Current } \\
(\mathrm{A})\end{array}$ & $\begin{array}{c}\text { Voltage } \\
(\mathrm{V})\end{array}$ & $\begin{array}{c}\text { Speed } \\
(\mathrm{mm} / \mathrm{sec})\end{array}$ \\
\hline $\begin{array}{c}\text { Flux-cored } \\
\text { arc welding }\end{array}$ & 3.0 & 280 & 28.5 & 16.5 \\
\hline
\end{tabular}

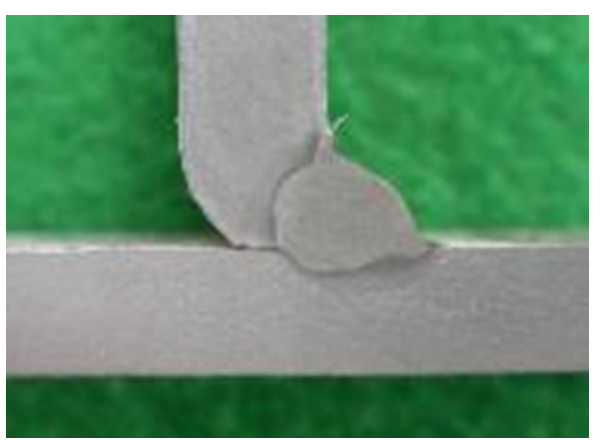

Fig. 3 Cross section of the one-sided fillet weld

interval on the top plate, a single-sided continuous fillet weld of a $3.0 \mathrm{~mm}$ thick throat is firstly laid on each stiffener with the welding condition listed in Table 1. Fig. 3 shows the macrograph of the fillet weld. The double-sided fillet welding to the transverse frame is subsequently followed as per the welding condition shown in Table 1.

After assembling the stiffeners and frames, the panel is turned over, and the butt welding between plates is accomplished in the flat position. Two types of the arc welding process listed in Table 2 were considered for 
Table 2 Butt welding conditions

\begin{tabular}{|c|c|c|c|c|}
\hline $\begin{array}{c}\text { Welding } \\
\text { Process }\end{array}$ & Groove & $\begin{array}{c}\text { Current } \\
(\mathrm{A})\end{array}$ & $\begin{array}{c}\text { Voltage } \\
(\mathrm{V})\end{array}$ & $\begin{array}{c}\text { Speed } \\
(\mathrm{mm} / \mathrm{sec})\end{array}$ \\
\hline SAW(Butt 1) & Square & 550 & 36 & 8.33 \\
\hline FCAW(Butt 2) & Single V & 320 & 34 & 10.0 \\
\hline
\end{tabular}

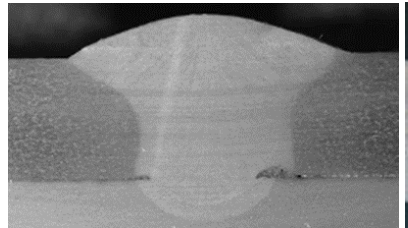

(a) Square groove

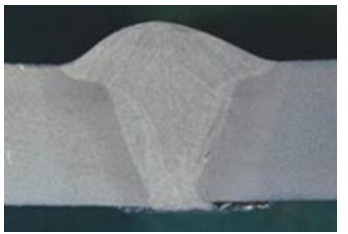

(b) Single V groove
Fig. 4 Macrographs of the butt welds

the single-sided butt weld with the stiffener backing. The welding conditions in Table 2 were determined through the qualification test to attain the complete penetration to the groove designed for each process. Fig. 4 shows the macrographs of the cross sections welded by the conditions in Table 2 . Complete penetration through the thickness of the plate is observed.

\subsection{Prediction scheme}

When a welded joint is long and continuous under a steady condition, every cross section of the weld normal to the welding direction experiences nearly the same time-dependent temperature transition except for a time lag in responses between cross sections examined. Provided a uniform joint constraints and a constant section geometry, stresses and strains in the weld can be represented on the plane of the cross section. The application of the two-dimensional (2D) model in the welding simulation can remarkably diminish the computational difficulty arising from the 3D model of the large welded structures. On the other hand, it has been shown that the welding residual stresses and distortion can be elastically estimated if the incompatible strains accumulated in the weld region are known ${ }^{12-14)}$. The elastic approach in the welding distortion analysis will much enlarge the size of the model to be solved in a real scale since the iterative non-linear structural behaviors can be linearly treated.

Michaleris et al. ${ }^{15)}$ proposed a two-step analysis procedure to predict the buckling distortion of welded thin plate panels. The 2D finite element model was adopted from the cross section of the weld to determine the transient temperature and residual stresses. The residual stress distribution calculated from the 2D model was used to derive a load pattern for the eigenvalue buck- ling prediction performed on the 3D panel model. Deng et al. ${ }^{16)}$ and Wang et al. ${ }^{17)}$ suggested another two-step approach to predict the welding-induced buckling distortion in a large thin plate panel. A series of $2 \mathrm{D}$ thermal elastic-plastic analyses were firstly implemented to obtain the inherent strain distributions individually from each cross section of the weld joints. From the first step, the inherent shrinkages were calculated through integrating the relevant component of the inherent strains over the cross section of the weld. The welding-induced buckling distortion was then elastically predicted by introducing the inherent shrinkages as the driving forces to the 3D panel model at the second step.

In this study, the 2D thermal elastic-plastic analysis was performed for the cross section model of the weld joint applied to the panel assembly to numerically derive the plastic strains in the weld, which is equivalent to the incompatible strains. As an instance of the 2D analysis, Fig. 5 illustrates the colored fringe pattern of the longitudinal plastic strain, $\varepsilon_{x}^{p}$, in the single-sided fillet weld of the $3 \mathrm{~mm}$ thick throat, and Fig. 6 shows the distribution of $\varepsilon_{x}^{p}$ along the line $\mathrm{AB}$ drawn in Fig. 5. The width of the plastic zone in the plate was about $31.0 \mathrm{~mm}$.

In order to evaluate the buckling sensitivity of the

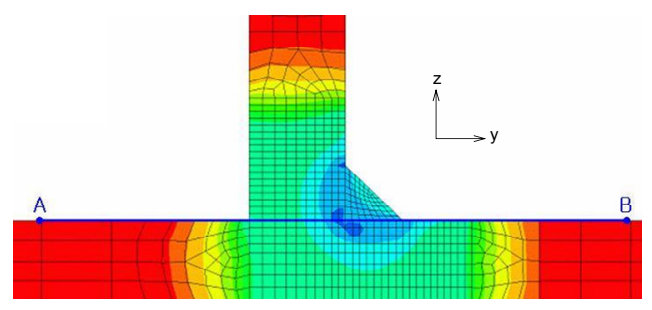

Fig. $5 \varepsilon_{x}^{p}$ in the fillet weld of $3 \mathrm{~mm}$ thick throat

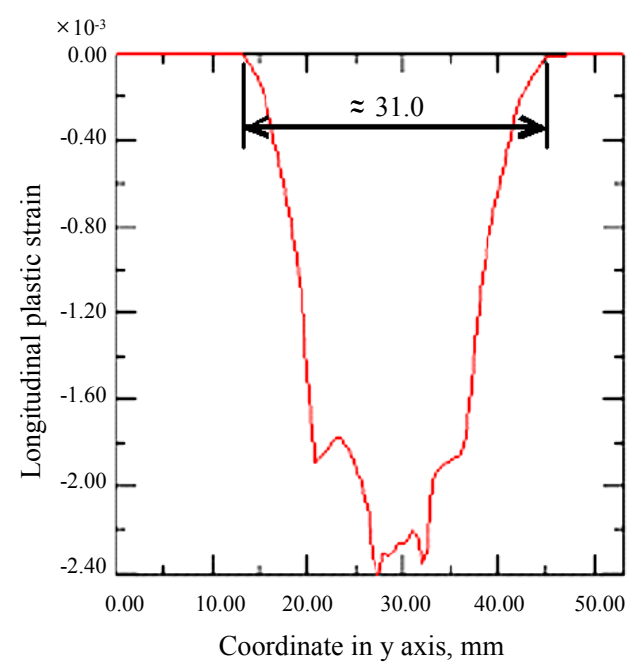

Fig. 6 Distribution of $\varepsilon_{x}^{p}$ along the line AB in Fig. 5 
welded panel, the eigenvalue analysis was carried out on the 3D panel model composed of 4-node shell elements. The perturbation load pattern $(\mathrm{T})$ for the eigenvalue analysis was determined from Eq. (1) where the normalized distribution of $\varepsilon_{x}^{p}$ through the thickness in the weld region, excluding the weld reinforcement, is divided by the plastic strain in the fusion zone $\left(\left(\varepsilon_{x}^{p}\right)_{F Z}\right)$. The letter, $h$, in Eq. (1) represents the plate thickness. The application of Eq. (1) over the cross section gives the load pattern distributed between 0 and 1.0 for each weld. The load pattern was applied as the thermal load distribution to the nodes of the corresponding weld joint in the 3D panel model.

$$
\mathbf{T}=\frac{\frac{1}{\mathbf{h}} \int \varepsilon_{\mathrm{x}}^{\mathbf{p}} \mathbf{d z}}{\left(\varepsilon_{\mathrm{x}}^{\mathbf{p}}\right)_{\mathrm{FZ}}}
$$

\subsection{Buckling sensitivity index (\%BSI)}

As a simple indicator of the buckling instability, the buckling sensitivity index (abbreviated to \%BSI) is defined as the percentage given by Eq. $(2)^{18,19)}$. The numerator in Eq. (2) represents the weld load in temperature scale, which is given by the fraction of $\left(\varepsilon_{x}^{p}\right)_{F Z}$ divided by the thermal expansion coefficient of the material. The denominator in Eq. (2) is the lowest eigenvalue from the analysis, which is equivalent to the buckling strength of the analyzed model. If the \%BSI by Eq. (2) exceeds 100 , it is the indication of the buckling instability. In the case of the \%BSI less than 100, a stable condition is prescribed.

$$
\% \mathrm{BSI}=\frac{\text { Weld Load }(\text { in temperature scale })}{\text { Buckling Strength }(\text { Lowest Eigenvalue })} \times 100
$$

\subsection{Buckling sensitivity of the welded panels}

Fig. 7 exemplifies the mode shape of the buckling distortion in the panel predicted by the eigenvalue analysis. In the analysis, the width of the free edge, w, was $345 \mathrm{~mm}$, and the welding condition of Butt 1 in Table 2

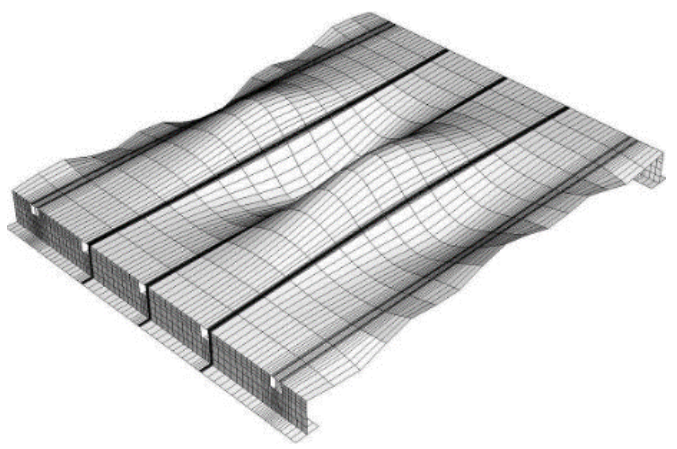

Fig. 7 Buckling mode at the lowest eigenvalue (\%BSI=112)

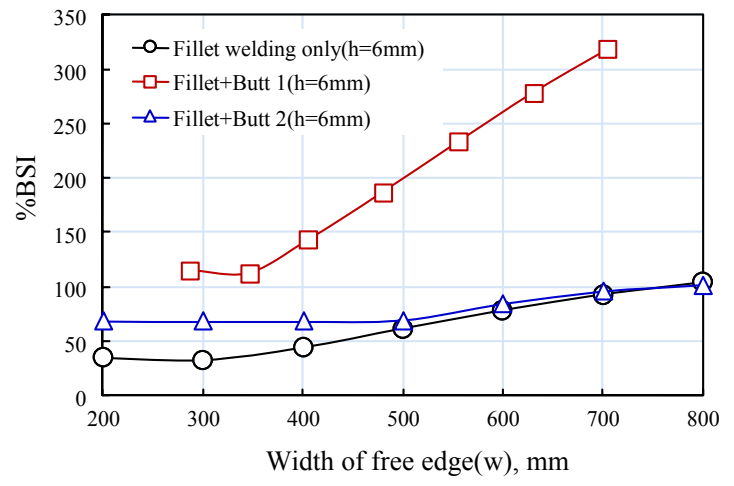

Fig. $8 \% \mathrm{BSI}$ as functions of the welding heat input and w

was considered for the butt joint. The \%BSI in this case was 112 , implying that the buckling instability is expected in the panel. The primary mode of buckling was generated in the interior of the panel whilst the higher mode prevailed in the region of the free edges.

Fig. 8 shows the variation of the buckling sensitivity of the panel as the functions of the welding heat input and the width of the free edge, w. Two butt welding conditions in Table 2 were respectively considered. The $\%$ BSI due to the fillet welding of the longitudinal stiffeners was also estimated. The \%BSI commonly increases as the free edge becomes wider in all welding conditions. At the fillet welding stage, the analysis estimated the \%BSI under 100 unless the width of the free edge is extended over $700 \mathrm{~mm}$. It implies that the plate will remain stable up to the $700 \mathrm{~mm}$ width of the free edge. For the analysis of the butt welding stage, the load patterns incurred due to both fillet and butt welding were simultaneously applied to the model. The behavior of the \%BSI is similar as the fillet welding stage where butt welding was conducted with the lower heat input, Butt 2, in Table 2. The result implies that the fillet welding will govern the buckling propensity when the butt joint can be designed to restrict the heat input under the certain level, and there exists the combination of the welding heat input and the width of the free edge to prevent the buckling distortion of the panel throughout the assembling process when the heat input in the butt weld is minimized. On the other hand, the buckling instability was expected regardless of the edge width if the higher heat input such as Butt 1 in Table 2 was given to the butt joint.

Fig. 9 illustrates the buckling sensitivity of the panel where the $7 \mathrm{~mm}$ thick plate was introduced in the analysis. Compared with the result of those for the $6 \mathrm{~mm}$ thick plate in Fig. 8, thickening by $1 \mathrm{~mm}$ further dropped the \%BSI so that the panel could be immune to buckling within the whole range of the free edge considered in the analysis. 


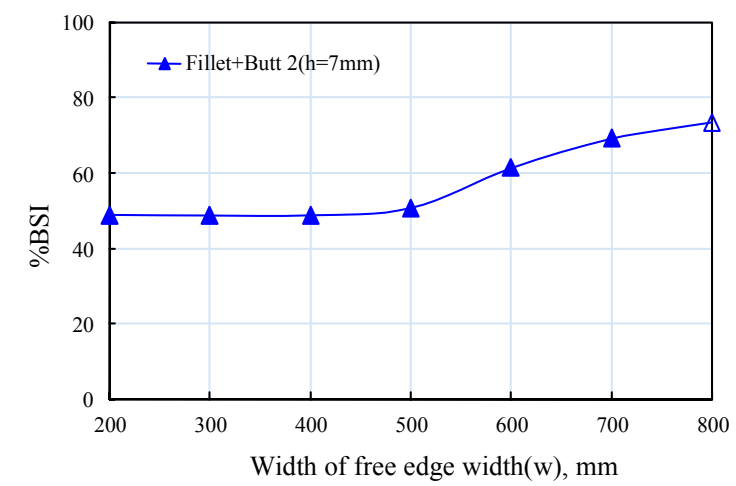

Fig. $9 \%$ BSI in $7 \mathrm{~mm}$ thick plated panels

From Fig. 8 and Fig. 9, it is clearly seen that the buckling sensitivity of the welded thin plate panels is influenced both by the structural design and welding variables, and thus the possibility of buckling in welded panels should be assessed not only from the point of the structural stiffness but from the view of the welding process adopted to the panel assembly. Further, the analytical scheme proposed in this study can be a proactive methodology to assess the fitness of the structural and welding design of the thin plate panels against buckling, which can be utilized in the early design stage.

\section{Experiments}

Experiments were performed to observe the welding distortion from the test panels. The configuration and dimension of the panel was the same as that illustrated in Fig. 1, specifically arranging the $400 \mathrm{~mm}$ width of the free edge equally at both sides. Fig. 10 shows the appearance of the test panel. The fillet welding condition in Table 1 was applied to the stiffener joints. Each of the welding conditions, Butt 1 and 2, in Table 2 was applied to the separate test panel.

Fig. 11 shows the morphological map of the out-of-plane distortion measured on the top surface of the panel welded by Butt 1 . As can be seen, the distortion prevailed mainly in the region of the free edges while a shallow wavy pattern was also shown in the inner region of the plate. It was revealed during the test that the distortion in the edge region increased after butt

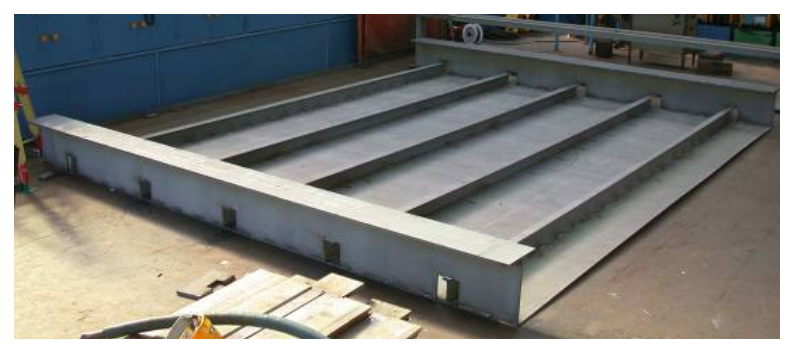

Fig. 10 Configuration of the test panel

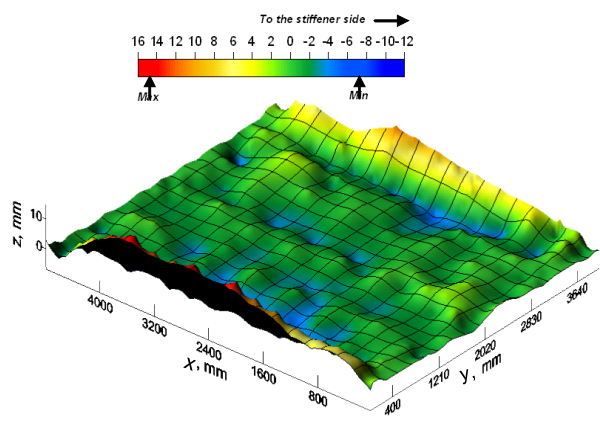

Fig. 11 Distortion measured from the test panel welded with the condition of butt 1

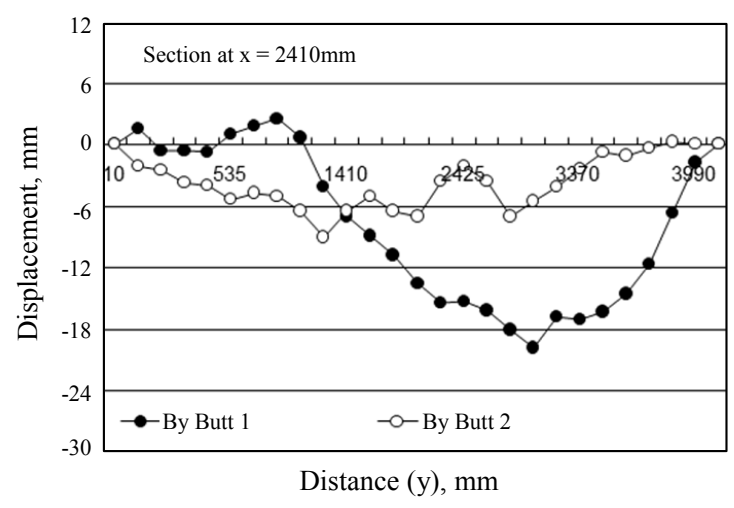

Fig. 12 Distortion meaured at the mid-length section

welding. Referring to the analysis result shown in Fig. 8, the buckling instability in the test panel is to be caused by butt welding. Furthermore, the shape of the distortion measured is in close proximity to the buckling mode shape predicted, as shown in Fig. 7. Therefore, it is reasonable to conclude that the distortion observed from the edge region of the test panel is produced by buckling of the plate caused during butt welding.

Fig. 12 compares the distortion measured at the mid-length cross section of the panels butt-welded by Butt 1 and 2 respectively. It is seen that the higher heat input to the butt joint induces the larger distortion in the thin plate. A half wavy patterns of the distortion in between longitudinal stiffeners, which might be the angular change caused by fillet welding, is seen in the panel welded by Butt 2. In the panel welded by Butt 1, the distortion tends to be an asymmetric full wave pattern with respect to the butt joint due to buckling of the plate.

\section{Conclusions}

In this study, a stepwise analytical scheme to predict the welding-induced buckling sensitivity in the thin plate panels was proposed. The eigenvalue analysis was adopted into the scheme to estimate the critical buckling state in the welding assembly of the thin plate panel. The 
perturbation load patterns for the analysis were derived from the longitudinal plastic strains in the welds, which were determined from the $2 \mathrm{D}$ thermal elastic-plastic analysis for the cross sections of the welds. In order to quantitatively evaluate the effect of design and welding parameters on the buckling sensitivity of the panels, the $\%$ BSI was defined as the indicator of whether or not the buckling instability might be expected due to a specific change in design and welding parameters. The effect of the plate thickness, the width of the free edge, the fabrication stage, and the welding heat input on the buckling instability was specifically investigated. If the distribution of longitudinal plastic strains in welds is acquired by any means, the proposed scheme can numerically estimate the buckling propensity of the welded thin plate panels.

To verify the buckling distortion predicted by the analysis, the welding assembly of the test panels was also carried out. The heat input in butt welding governed the buckling region and the buckling mode shape in the test panels. Higher heat input might cause the buckling instability, and thus leave the higher magnitude of the distortion in the region of the free edges. Through the experiments, the prediction scheme proposed in this study has been reasonably validated.

ORCID: Myoung Soo Han: https://orcid.org/0000-0003-4383-4817

\section{References}

1. C. Conrardy and R. Dull, Control of Distortion in Thin Ship Panels, J. Ship Prod. 13(2) (1997) 83-92.

2. C. Conrardy, T. D. Huang, D. Harwig, P. Dong, L. Kvidahl, N. Evans and A. Treaster, Practical Welding Techniques to Minimize Distortion in Lightweight Ship Structures, J. Ship Prod. 22(4) (2006) 239-247.

3. C. L. Tsai and M. S. Han, Thermal and Mechanical Evolution of Welding-induced Buckling Distortion, $J$. Chinese Inst. Eng. 27(6) (2004) 907-920. https://doi.org/10.1080/02533839.2004.9670943

4. C. L. Tsai, M. S. Han and G. H. Jung, Investigating the Bifurcation Phenomenon in Plate Welding, Weld. J. 85 (7) (2006) 151s-162s.

5. K. Masubuchi, Prediction and Control of Residual Stresses and Distortion in Welded Structures, Trans. JWRI, 25(2) (1996) 53-67.
6. C. L. Tsai, S. C. Park and W. T. Cheng, Welding Distortion of a Thin-Plate Panel Structure, Weld. J. 78 (1999) 156-s-165-s.

7. D. Deng, H. Serozawa and H. Murakawa, Prediction of Welding Distortion during the Assembly Process of Thin Plate Structures(2 ${ }^{\text {nd }}$ Report)-Influence of Welding Sequence on Welding Distortion, J. Kansai Soc. N. A. 242 (2004) 97-104.

8. Q. Guan, R. H. Leggatt and K. W. Brown, Low Stress Non-distortion(LSND) TIG Welding of Thin-walled Structural Elements, TWI Research Report 374, The Welding Institute, Cambridge, UK (1988).

9. P. Michaleris and X. Sun, Finite Element Analysis of Thermal Tension Techniques Mitigating Weld Buckling Distortion, Weld. J. 76(11) (1997) 451-s to 457-s.

10. F. Roland, T. Reinert and G. Pethan, Laser Welding in Shipbuilding - An Overview of the Activities at Meyer Werft, Proc. IIW, Copenhagen, Denmark (2002).

11. F. Roland and H. Lembeck, Laser Beam Welding in Shipbuilding-Experience and Perspectives at Meyer Shipyard, Proceeding of 7th International Aachen Welding Conference, Aachen, Germany (2001) 463-475.

12. Y. Ueda, K. Nakacho and K. Moriyama, Simple prediction methods for welding deflection and residual stress of stiffened panels, Trans. JWRI, 15(2) (1986) 197-204.

13. Y. Ueda and M. G. Yuan, Prediction of residual stresses in butt welded plates using inherent strains, Transactions of the ASME, J. Eng. Mater. Technol. 115(10) (1993) 417-423.

14. X. M. Zhong, H. Murakawa and Y. Ueda, Buckling behavior of plates under idealized inherent strain, Trans. JWRI, 24(2) (1995) 87-91.

15. D. A. Michaleris and A. DeBiccari, Prediction of welding distortion, Weld. J. 76(4) (1997) 172s-180s.

16. D. Deng, N. Ma, H. Murakawa, Finite Element Analysis of Welding Distortion in a Large Thin-plate Panel Structure, Trans. JWRI, 40(1) (2011) 89-100.

17. J. Wang, S. Rashed, H. Murakawa and M. Shibahara, Investigation of Buckling Deformation of Thin Plate Welded Structures, Proceedings of the 21st ISOPE, Hawaii, USA (2011) 125-131.

18. M. S. Han, Welding-induced Distortion and Effective Straightening Practices in Thin-walled Structures, Proceedings of the 2013 Autumn Annual KWJS Conference, Busan, Korea (2013) 45.

19. M. S. Han and H. U. Kim, Practical Measures to Minimize Welding-induced Distortion in Thin-Plated Panels, Proceedings of the 2008 Autumn Annual KWJS Conference, Incheon, Korea (2008) 108. 